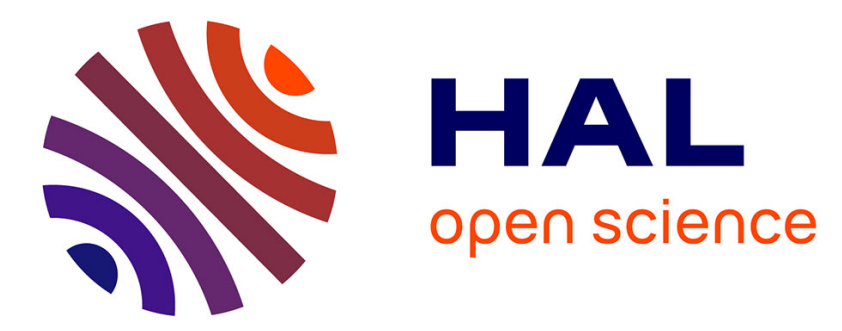

\title{
Calibration of a hydraulic architecture-based growth model of cotton plants
}

Philippe de Reffye, Frédéric Blaise, Stéphane Chemouny, Sylvie Jaffuel, Thierry Fourcaud, François Houllier

\section{- To cite this version:}

Philippe de Reffye, Frédéric Blaise, Stéphane Chemouny, Sylvie Jaffuel, Thierry Fourcaud, et al.. Calibration of a hydraulic architecture-based growth model of cotton plants. Agronomie, 1999, 19 (3-4), pp.265-280. hal-00885929

\section{HAL Id: hal-00885929 https://hal.science/hal-00885929}

Submitted on 1 Jan 1999

HAL is a multi-disciplinary open access archive for the deposit and dissemination of scientific research documents, whether they are published or not. The documents may come from teaching and research institutions in France or abroad, or from public or private research centers.
L'archive ouverte pluridisciplinaire HAL, est destinée au dépôt et à la diffusion de documents scientifiques de niveau recherche, publiés ou non, émanant des établissements d'enseignement et de recherche français ou étrangers, des laboratoires publics ou privés. 
(C) Inra/Elsevier, Paris

\title{
Original article
}

\section{Calibration of a hydraulic architecture-based growth model of cotton plants}

\author{
Philippe de Reffye ${ }^{\mathrm{a}, \mathrm{b}}$, Frédéric Blaise ${ }^{\mathrm{a}, \mathrm{b}}$, Stéphane Chemouny ${ }^{\mathrm{a}}$, Sylvie Jaffuel ${ }^{\mathrm{a}}$, \\ Thierry Fourcaud ${ }^{\mathrm{a}, \mathrm{c}}$, François Houllier ${ }^{\mathrm{a} *}$ \\ a Programme modélisation des plantes (AMAP), centre de coopération internationale en recherche agronomique \\ pour le développement (Cirad), Campus de Baillarguet, BP 5035, 34032 Montpellier cedex 1, France \\ b Laboratoire franco-chinois d'informatique, d'automatique et de mathématiques appliquées (LIAMA), \\ Institute of Automation, Chinese Academy of Sciences, PO Box 2728, Beijing 100080, China \\ ' Laboratoire de rhéologie du bois de Bordeaux, UMR 123 CNRS, Inra, université Bordeaux 1, \\ domaine de l'Hermitage, BP 10,33610 Cestas Gazinet, France
}

(Received 10 November 1998; accepted 22 February 1999)

\begin{abstract}
The AMAPpara model describes long-term plant growth as the cumulative output of the cyclic interactions between plant ecophysiological functioning and architectural development. It is based on the classical relationship between transpiration and biomass production, under the assumption of a constant water use efficiency. Biomass increment is allocated to organs (leaves, inter-nodes) according to their sink strength and their expansion laws. Allometric rules are used to derive the geometry of the organs as a function of their volume or biomass. Organ geometry determines the hydraulic architecture of the plant, from which water transpiration is computed. Feedback between plant growth and architecture is modelled through a recurrence equation which links the successive growth cycles to each other. Under the assumption that climatic conditions are approximately stable over the whole growth period, the parameters of AMAPpara can be estimated from the observation of plant architecture and morphology at the end of their growth. A preliminary calibration of the model was carried out for cotton, and yielded quantitatively and qualitatively satisfactory results. (C) Inra/Elsevier, Paris.)
\end{abstract}

\section{modelling / plant architecture / hydraulic architecture / growth / simulation}

Résumé - Calibration d'un modèle de croissance du cotonnier. Le modèle AMAPpara décrit la croissance à long terme de plantes comme le résultat cumulatif des interactions cycliques entre leur développement architectural et leur fonctionnement écophysiologique. Ce modèle est basé sur la relation classique constatée entre la transpiration et la production de biomasse; l'efficience d'utilisation de l'eau y est supposée constante. L'accroissement en biomasse est par-

Communicated by Gérard Guyot (Avignon, France)

* Correspondence and reprints

houllier@cirad.fr 
tagé entre les différents organes (feuilles, entre-nœuds) selon la force de leur puits et selon leur loi d'expansion. Leur forme est calculée selon des règles géométriques établies empiriquement. On en déduit l'architecture hydraulique puis la transpiration sous l'action d'un potentiel hydrique. Une équation de récurrence est ainsi établie : elle relie automatiquement la croissance et l'architecture au cours des cycles de croissance successifs.

Sous l'hypothèse que les conditions climatiques oscillent faiblement autour de valeurs moyennes stables durant tout le cycle de croissance, il est possible d'estimer les paramètres de AMAPpara à partir de données architecturales et morphologiques mesurées en fin de croissance. Une application est faite sur cotonnier et l'on obtient un ajustement satisfaisant du modèle qui fournit des prédictions qualitativement et quantitativement satisfaisantes de l'architecture de la plante. (@) Inra/Elsevier, Paris.)

\section{modélisation / architecture des plantes / architecture hydraulique / croissance / simulation}

\section{Introduction}

Most of the quantitative process-based models of plant growth [19] do not take plant architecture into account. These models compute the dry matter production from physical parameters (e.g. temperature, light) or morphological indexes (e.g. leaf area index (LAI)). In such models, there is no feedback between plant structure and plant functioning. This fact is now considered as a strong limitation of these models (see for example the two recent international workshops on structure-function tree models organized in Helsinki (1996) and Clermont-Ferrand (1998)).

On the other hand, computer graphics scientists have recently tried to generate realistic shapes of plants by simulating morphogenetic and geometric rules described by botanists. Although they do not include any physiological knowledge, software based on L-systems [13], automaton [17] and graph descriptions [10] have demonstrated their capability to generate virtual 3D plant mock-ups. Such pure morphogenetic simulators can be calibrated and validated, with a good agreement between the real and simulated plant structure [12]. However, these models cannot account for the interactions between plant growth and plant architecture, even if some of them (e.g. Grogra [9], Lignum [11], L-system [6]) begin to integrate ecophysiological rules and to deal with the allocation of the assimilates inside the plant structure. 3D mock-ups can also be used for computing light interception [5] or water transpiration [14], or for describing competition for space within a forest stand $[3,9,13]$.
The time step in process-based models which take leaf physiology into account is usually short: from a few minutes to several hours. Light interception and $\mathrm{CO}_{2}$ assimilation, which depend on biophysical parameters and on stomatal opening, are indeed measured and simulated at a short time step [14]. If we consider the long-term architectural development and growth of the whole plant (i.e. over plant life span or crop cycle), longer time steps seem more appropriate. For example, it has been shown that the cumulative sum of temperatures is sufficient to predict the number of new metamers produced by a terminal meristem. As a consequence, the total number of organs in a given plant (i.e. the topological structure) does not depend on the local variations but on the cumulative effect of the physical environment.

Furthermore, at the plant level and over long periods, the amount of biomass produced is usually well correlated with the cumulative water transpiration, although this may be untrue for short periods. The 3D geometrical architecture is not taken into account in this general result. However, Ryan and Yoder [18] argue that the hydraulic resistance to transpiration, which is closely linked to plant architecture, is the major factor that limits longterm tree growth.

The basic underlying idea of the AMAPpara model is thus to simultaneously consider the topological and geometrical structures of the plant, and to relate them to the environmental conditions: the number of new organs is predicted from the cumulative temperatures according to the architectural model; the volume and geometry of each organ are computed according to biomass production and 
allocation, using sink-source and allometric concepts.

The aim of ecophysiology is to monitor at short time steps how plant growth interacts with environmental conditions (e.g. the Tomgro [7] or Gossym [1] models). However, the architectural point of view is also important and has not yet been developed in this context. Which conditions was a plant subjected to during its life span in order to explain the observed architecture? This is a common question, notably in forestry, which can be answered by considering jointly the topological and geometrical structure of the plant [15].

The theoretical approach developed in AMAPpara [16] deals with these two points of view. In this paper, we focus on the architectural development of a plant, and on how it can be related to water transpiration and the concept of hydraulic architecture, to biomass production and the concept of water use efficiency (WUE), to biomass allocation and the concept of sink strength. Up to now, no experimental validation of this model had been performed. This paper presents the first attempt to calibrate the model, i.e. to estimate its parameters from experimental data. Our goal is thus to match the predicted plant geometry with the observed architecture of cotton plants.

\section{Materials and methods}

In order to facilitate the comparisons between observations and model predictions, a field experiment was conducted, with the aim to have a very simple plant architecture. Cotton plants were planted at a low density $(1 \mathrm{~m} \times 4 \mathrm{~m})$ and were regularly irrigated. Thus no competition and no hydric stress were expected. Plants were continuously monitored and branches were pruned immediately after their initiation, so that the plants consisted of a single stem. Control plants were not pruned, but all the flowering buds were removed in order to restrict plant development to a pure vegetative growth.

At the end of the growth season, the plants were felled and exhaustively measured: the length and diameter of each inter-node, as well as the size and weight of the petiole and of the blade of each leaf were measured. Lengths and diameters were expressed in centimetres and weights in grams.
The effect of pruning was spectacular (figure 1): tall single stems with big leaves and long inter-nodes were obtained, but their biomass was less than the biomass of the control (unpruned) plants. The mean height and weight were, respectively, $2.1 \mathrm{~m}$ and $1.1 \mathrm{~kg}$ for the pruned plants versus $1.5 \mathrm{~m}$ and $1.8 \mathrm{~kg}$ for the control plants. The number of stem nodes was 35 for pruned plants versus 25 for control plants.

The growth of the pruned cotton-trees was significantly (quantitative) related to the sum of temperatures (figure 2). Climatic data were available for temperature, solar radiation and potential evapotranspiration (ETP in $\mathrm{mm}_{\mathrm{day}}{ }^{-1}$ ). These data were plotted against time, using either a fixed 4-day time step, or a variable time step defined by the delay between the initiation of two consecutive metamers by the apical bud (figure 3). The latter time step was closely related to the phyllochrone and to the leaf initiation rate, which varied from 7 days (early May) to 3 days (July).

When the fixed time step was used, we observed growth trends over time (figure $3 a, c$ ). These trends vanished when the variable time step was used fig-
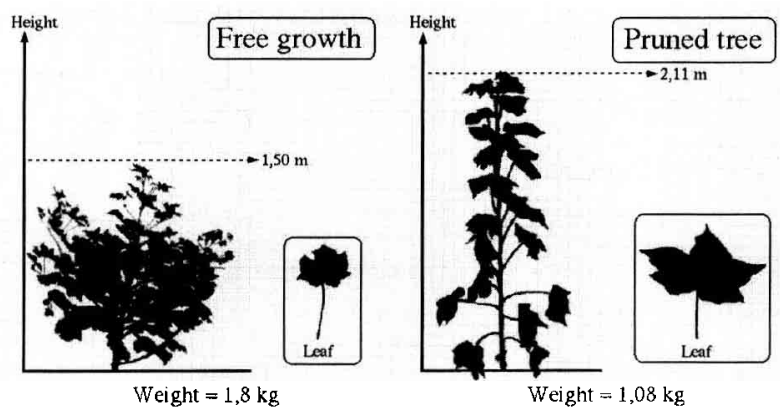

Figure 1. Photograph of control (unpruned) and pruned cotton plants.

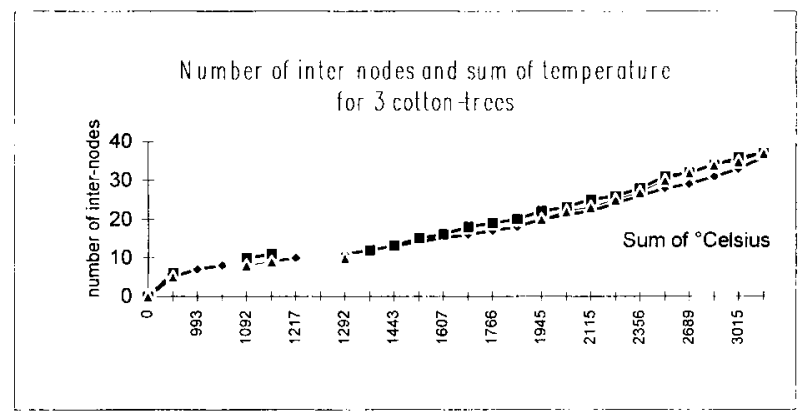

Figure 2. Relationship between growth rate and the sum of temperatures for pruned cotton plants. 

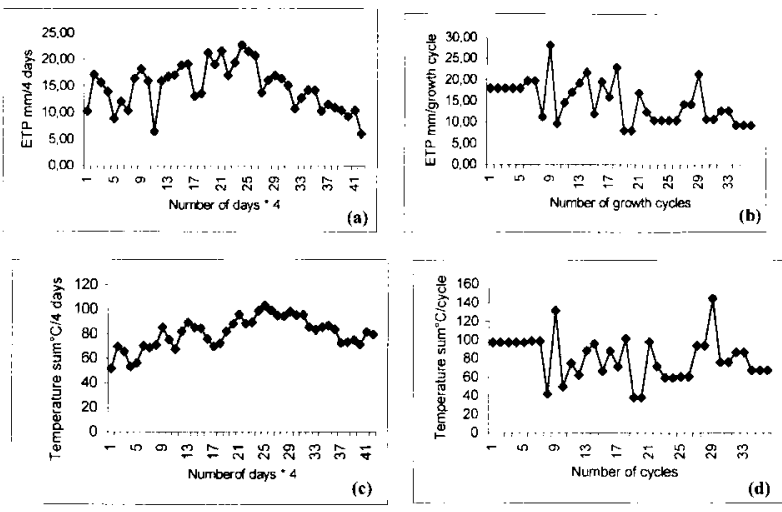

Figure 3. Evolution of the cumulated temperatures and cumulated potential evapotranspiration ETP every 4 days or during a growth cycle over the period of growth of the cotton plants.

ure $3 b, d)$. When the temperature increased, the growth rate also increased, so that the sum of temperatures per growth cycle remained approximately constant. With ETP, which represents the climatic water demand, we observed the same phenomenon. These results indicate that the amount of energy received by a plant during a growth cycle randomly fluctuated around a mean constant value. We therefore chose to use the leaf initiation rate as the basic time unit.

\section{The mathematical model AMAPpara}

\subsection{Biological assumptions}

We consider a theoretical plant made up of fresh matter, which contains approximately $80 \% \mathrm{H}_{2} \mathrm{O}$ and $15 \%$ assimilates $\left(\mathrm{CO}_{2}+\mathrm{H}_{2} \mathrm{O}\right)$. Water is always linked in the same proportion as the carbon, and represents $90 \%$ of the fresh matter and with $\mathrm{CO}_{2} 95 \%$ of plant structure. This is quite sufficient to control the allocation of matter in the architecture. The relative density is set to $1.0 \mathrm{~g} . \mathrm{cm}^{-3}$ (strictly speaking, this value is only true for succulent young organs). Only the aerial part of the plant is taken into consideration, and its weight is supposed to be always proportional to the weight of the root system.
We consider two types of organs, which result from either primary or secondary growth: leaves which consist in a petiole and a blade, and internodes which are made up of a pith and of growth 'layers', which result from cambial activity. Primary growth consists in adding new metamers (apical growth), but also in the lengthening of the existing metamers: leaf and inter-node expansion are indeed supposed to last over several cycles. The case of inter-nodes is special: i) inter-node lengthening and inter-node pith thickening occur during the first cycles just after inter-node initiation; ii) secondary growth adds a new external layer at each cycle (in the case of trees under a marked seasonal climate, these layers are visible and are termed growth rings), thus increasing the diameter of the inter-nodes.

The blade of the leaves is supposed to have a constant thickness so that their area is proportional to their volume. The biomass ratio between leaf lamina and petiole is also supposed to be constant during leaf growth. Petioles and inter-nodes are described as cylinders whose geometry depends on allometric rules: the long-term ratio between their cross-sectional area and their length is expressed as a function of their fresh matter biomass.

\subsection{Architectural assumptions}

The plant is supposed to grow on a cyclic basis: here, for pruned cotton plants, growth cycle is considered to be the time step between the initiation of two successive leaves along the stem (for temperate tree species growing over many years, we would probably use a longer growth cycle, such as one year). Plant age (respectively, leaf age) is thus expressed as the number of growth cycles since the plant germinated (respectively, since the leaf appeared).

For a single stemmed cotton plant, there is only one leaf for a given age (measured as a number of growth cycles) and the metamers of the stem are noted with double indices: $j$ for metamer rank along the stem (counted upwards), $i$ for plant age (with $i \geq j$ ). For example: $q_{a}(i, j)$ is the biomass of 
the $j$ th leaf above the ground at plant age $i$; leaf age is $k=i-j+1$.

At the beginning of a growth cycle, new organs are created while the existing organs further expand; both new and existing organs use the reserve of available fresh matter produced in earlier cycles. During the cycle, plant architecture remains constant and leaves produce fresh matter. At the end of the cycle, secondary growth (i.e. growth layers) occurs and is simulated using the allocation and allometric rules. The remaining fresh matter is stored as a reserve for the next cycle.

\subsection{Ecophysiological assumptions}

It is known that about $600 \mathrm{~L}$ of water are needed in order to produce $1 \mathrm{~kg}$ of dry matter of potatoes (or $10 \mathrm{~kg}$ of fresh matter). Here, we consider the case where there is no hydric stress, and suppose that there is a proportionality between water transpiration and fresh matter produced during a growth cycle. During the $i$ th growth cycle occurring at date $t_{i}$, the net quantity of fresh matter $Q f m(i)$ produced by the plant is given by the classical formula:

$$
Q f m(i)=\frac{K e\left(t_{i}\right) \cdot \Delta \Psi\left(t_{i}\right)}{R\left(t_{i}\right)}
$$

where $\Delta t_{i}$ is the duration of the cycle; $\Delta \Psi$ is the hydric potential; $K e$ is the WUE, which depends on biophysical parameters, i.e. light, temperature, and implicitly includes a respiration term; $R$ is the hydraulic resistance of the plant.

These variables and parameters are supposed to maintain their average value during the growth cycle so that, in the first approximation, the product $E=K e \cdot \Delta \Psi \cdot \Delta t$ is constant during a cycle. This product is defined as the potential of fresh matter production. The duration of each growth cycle is adjusted so that $E$ remains constant from one cycle to the other (see section 1): bad climatic conditions result in longer periods for the cycle, whereas good conditions result in shorter growth cycles. Equation (1) can thus be simplified into equation (2):

$$
Q f m(i)=\frac{E}{R(i)}
$$

In this paper, we consider that the hydraulic resistance of the plant depends on the resistance of the leaves $R_{a}$ alone. Discounting shading effects, which have a marked influence on leaf temperature and, therefore, on transpiration, each leaf is supposed to receive the same amount of energy and to undergo the same climatic demand (on a per leaf area basis), so that equation (2) can be written as:

$$
Q f m(i)=\sum_{j=1}^{N(i)} \frac{E}{R_{a}(i, j)}
$$

where $N(i)$ is the total number of leaves at growth cycle $i$ (if some leaves are dead, the summation starts at the first living leaf). The numerator is constant $(E)$, but the denominator $\left(R_{a}(i, j)\right)$ varies from one leaf to another.

Leaf resistance is the sum of blade resistance and petiole resistance. For blade resistance, we consider the total resistance needed for the water to pass through the leaf surface and to change from liquid to gaseous state. This is convenient from the architectural point of view (from the ecophysiological point of view, this global resistance should be further detailed). Therefore, leaf blade resistance can be expressed as:

$$
r_{a 1}=\rho_{a 1} \frac{\varepsilon}{S}
$$

where $\rho_{a 1}, S$ and $\varepsilon$ are the resistivity, the area and the thickness of the blade, respectively.

The resistance of the petiole is:

$$
r_{a 2}=\rho_{a 2} \frac{l}{\sigma}
$$

where $\rho_{a 2}, \sigma$ and $l$ are the resistivity, the area and the length of the petiole, respectively.

Because of the leaf expansion process, the dimensions of the organs $(S(i, j), l(i, j), \sigma(i, j))$ 
depend on their rank along the stem $(j)$ and on the cycle number $(i)$. Considering that a growth cycle covers a short time step, the parameters $\rho_{\mathrm{a} 1}, \rho_{\mathrm{a} 2}$ are supposed to be constant during a growth cycle. As for $E$, we further assume that these parameters do not change from one cycle to the other (this assumption could be relaxed, using knowledge on the relationship between $\rho_{\mathrm{a} 1}, \rho_{\mathrm{a} 2}$ and the environmental conditions). The total resistance of a leaf is therefore:

$$
R_{a}(i, j)=\rho_{a 1} \frac{\varepsilon}{S(i, j)}+\rho_{a 2} \frac{l(i, j)}{\sigma(i, j)}
$$

Under these assumptions, at cycle $i$, the production of fresh matter depends only on the number of leaves that are available for photosynthesis. We define the parameter $t_{a}$ as the life span of the leaves. The fresh matter increment during the $i$ th growth cycle is:

$$
Q f m(i)=\sum_{j=\max \left(1, i-t_{a}\right)}^{i} \frac{E}{\rho_{a 1} \frac{\varepsilon}{S(i, j)}+\rho_{a 2} \frac{l(i, j)}{\sigma(i, j)}}
$$

Although the parameter $E$ is assumed to be constant, the biomass production of a leaf changes from one cycle to the other: this is due to the fact that leaf expansion occurs over several cycles and thus modifies blade and petiole size, and consequently their hydraulic resistance.

From the architectural point of view, only the ratios $E / \rho_{a 1}$ and $E / \rho_{a 2}$ have to be known to simulate plant development; these ratios can be estimated from experimental data. If we set $E$ to an arbitrary value (e.g. $E=1000$ ), the parameters $\rho_{a 1}$ and $\rho_{a 2}$ can be adjusted at proper values to fit well the data (see section 3.1.2). Changing the arbitrary value of $E$ would result in a proportional change of the estimated values of $\rho_{a 1}$ and $\rho_{a 2}$, but the estimated ratio $\rho_{a 1} / \rho_{a 2}$ would remain constant.

\subsection{Modelling biomass allocation and organ expansion}

Biomass increment contributes to both primary and secondary growth. Primary growth consists in adding new leaves and inter-nodes, but also in leaf and inter-node expansion which lasts over several cycles; during leaf expansion, we assume that biomass increment is shared, with a constant ratio, between leaf blade and leaf petiole. Secondary growth only concerns the addition of external growth layers on the stem: in this paper, we suppose that, for a given growth cycle, the distribution of the biomass increment along the stem is uniform, i.e. the vertical profile of the cross-sectional area of a given growth layer is constant.

Biomass increment is hierarchically allocated to the organs: i) it is shared among three primary compartments, also named primary sinks: the leaf compartment, the inter-node compartment and the layer compartment; ii) within each compartment, the matter is further distributed among secondary sinks represented by the various organs, each of them being at a given stage of expansion. Various models belonging to this family of allocation models can be built. Here, we present one member of this family.

\subsubsection{Primary sinks}

For the $i$ th cycle $(1 \leq i \leq \mathrm{N})$, we define:

- $Q(i)$ as the fresh matter produced by the plant during the cycle $i$;

- $Q_{a}(i)$ as the fresh matter allocated to the leaf compartment;

- $Q_{e}(i)$ as the fresh matter allocated to the internode compartment;

- $Q_{c}(i)$ as the fresh matter allocated to the layer compartment;

- $p_{a}$ as the relative sink strength of the leaf compartment;

$-p_{e}$ as the relative sink strength of the inter-node compartment;

$-p_{c}$ as the relative sink strength of the layer compartment.

The parameters $p_{a}, p_{e}$ and $p_{c}$ are supposed to be constant during growth and to verify the constraint $p_{a}+p_{e}+p_{c}=1$. We have: $Q_{a}(i)=p_{a} \cdot Q(i)$, $Q_{e}(i)=p_{e} \cdot Q(i)$ and $Q_{c}(i)=p_{c} \cdot Q(i)$. 
Note that leaves simultaneously play the roles of biomass source and biomass sink.

\subsubsection{Secondary sinks}

We further define the leaf expansion function: $f_{a}(k)$ where $k$ is the age of the leaf expressed in number of growth cycles $\left(1 \leq k \leq t_{a}\right)$. This function behaves as a probability distribution function and verifies the constraint $\Sigma_{k=1}^{t a} f_{a}(k)=1$ (i.e. a leaf fully expands before dying). Both $t_{a}$ (the leaf life span) and $f_{a}(k)$ are supposed to be the same for all leaves.

The leaf expansion function is related to the physiological maturation of a leaf and measures its capacity to further grow. If $\widetilde{Q}(k)$ is the quantity of fresh matter available in the local environment of a $k$ cycle-aged leaf, the fresh matter increment of this leaf is: $f_{a}(k) \cdot \widetilde{Q}(k)$. The volume of a leaf of a $i$ cycle-aged leaf cycle is thus: $\Sigma_{k=1}^{i} f_{a}(k) \cdot \widetilde{Q}(k)$. If $f_{a}(k)=0$ beyond a given value of $k$, then the leaf is no more a sink, but it can still be a source until it reaches age $t_{a}$.

In this model, leaf growth depends on both the leaf expansion function and the availability of assimilates (or fresh matter): multiple combinations can therefore yield the same final result. The distribution $f_{a}(k)$ is hidden and cannot be directly measured.

In this paper, we choose to model leaf (but also inter-node and layer) expansion function with negative binomial distributions truncated at 12 growth cycles. The second parameter of the negative binomial distributions is arbitrarily set to 2 . For a leaf of age $k\left(1<k<t_{a}\right)$, the value of the expansion function is therefore:

$$
f_{a}(k)=\frac{C_{k}^{k-1} \cdot b n_{a}^{k-1} \cdot\left(1-b n_{a}\right)^{2}}{\sum_{i=1}^{t a} C_{i}^{i-1} \cdot b n_{a}^{i-1} \cdot\left(1-b n_{a}\right)^{2}}
$$

where $b n_{a}$ is an unknown parameter (to be estimated).

\subsection{Modelling growth as a recurrent phenomenon}

Plant functioning and development can be modelled by a recurrence relationship from one cycle to the next. Plant growth starts with the reserve $Q(0)$ (expressed in fresh matter), which is stored in the seed.

\subsubsection{Leaf growth}

At the $i$ th growth cycle, the biomass of the $j$ th leaf $(1 \leq j \leq i)$ is noted $q_{a}(i, j)$.

Cycle 1: the quantity of fresh matter that goes into the first leaf is: $q_{a}(1,1)=f_{a}(1) \cdot Q_{a}(0)$. The quantity $\widetilde{Q}_{a}(1)=\left(1-f_{a}(1)\right) \cdot Q_{a}(0)$ is not consumed during this cycle and is stored in the plant as a reserve for cycle 2 .

Cycle 2: during cycle 1, photosynthesis produces the quantity $Q(1)$ of fresh matter, so that the amount of reserves available to build the second leaf and to ensure the expansion of the first leaf is: $\widetilde{Q}_{a}(1)+Q_{a}(1)$. The following system can be written:

$$
\left\{\begin{array}{l}
q_{a}(2,1)=f_{a}(1) \cdot Q_{a}(0)+f_{a}(2) \cdot\left(\tilde{Q}_{a}(1)+Q_{a}(1)\right) \\
q_{a}(2,2)=f_{a}(1) \cdot\left(\tilde{Q}_{a}(1)+Q_{a}(1)\right)
\end{array}\right.
$$

The quantity $\widetilde{Q}_{a}(2)=\left(\widetilde{Q}_{a}(1)+Q_{a}(1)\right) \cdot\left(1-f_{a}(1)\right.$ $\left.-f_{a}(2)\right)$ is not consumed during this cycle, and is stored in the plant as a reserve for the third cycle. A recurrence relationship thus appears between the successive quantities of fresh matter produced by the plant. This system can be formally described by the matrix equation:

$$
\left.\left[\begin{array}{l}
q_{a}(2,1) \\
q_{d}(2,2)
\end{array}\right]=\left[\begin{array}{cc}
f_{a}(1) & f_{a}(2) \\
0 & f_{a}(1)
\end{array}\right] \cdot \mid \begin{array}{cc}
1 & 0 \\
1-f_{a}(1) & 1
\end{array}\right] \cdot\left[\begin{array}{l}
Q_{d}(0) \\
Q_{d}(1)
\end{array} \mid\right.
$$

Cycle 3: the matrix recurrence equation can be written:

$$
\left[\begin{array}{l}
q_{d}(3,1) \\
q_{a}(3,2) \\
q_{a}(3,3)
\end{array}\right]=\left[\begin{array}{ccc}
f_{i}(1) & f_{a}(2) & f_{h}(3) \\
0 & f_{a}(1) & f_{a}(2) \\
0 & 0 & f_{a}(1)
\end{array} \mid\right.
$$




$$
\left|\begin{array}{crr}
1 & 0 & 0 \\
1-f_{i}(1) & 1 & 0 \\
\left(1-f_{d}(1)\right)\left(1-f_{i}(1)-f_{a}(2)\right) & 1-f_{a}(1)-f_{a}(2) & 1
\end{array}\right| \cdot\left|\begin{array}{c}
Q_{d}(0) \\
Q_{d}(1) \\
Q_{a}(2)
\end{array}\right|
$$

This matrix system links leaf biomass at each rank along the stem to the amount of fresh matter available in the leaf compartment at each growth cycle. The quantity of fresh matter that is not used according to the leaf expansion function is stored as a reserve for the next cycle.

\subsubsection{Inter-node growth}

As for leaves, we define the expansion function for inter-node pith $\left(f_{e}(k)\right)$. The quantity $q_{e}(i, j)$ refers to the biomass of the pith of the inter-node located at rank $j$ along the stem when plant age is $i$. The inter-node compartment contains the amount $Q_{e}(i)$ of fresh matter. The initial amount of fresh matter allocated from the seed to the inter-node compartment is $Q_{e}(0)=p_{e} \cdot Q(0)$. At cycle 3 , the recurrence equation is:

$$
\begin{aligned}
& \left|\begin{array}{l}
q_{e}(3,1) \\
q_{e}(3,2) \\
q_{e}(3,3)
\end{array}\right|=\left|\begin{array}{ccc}
f_{e}(1) & f_{e}(2) & f_{e}(3) \\
0 & f_{e}(1) & f_{e}(2) \\
0 & 0 & f_{e}(1)
\end{array}\right| . \\
& \left|\begin{array}{crr}
1 & 0 & 0 \\
1-f_{e}(1) & 1 & 0 \\
\left(1-f_{k}(1)\right)\left(1-f_{e}(1)-f_{e}(2)\right) & 1-f_{e}(1)-f_{e}(2) & 1
\end{array}\right| \cdot\left|\begin{array}{l}
Q_{c}(0) \\
Q_{e}(1) \\
Q_{e}(2)
\end{array}\right|
\end{aligned}
$$

\subsubsection{Secondary growth: adding layers on the stem}

For the layers, there are differences due to the fact i) that $l$ th layer (i.e. the layer laid down at the end of cycle $l$ ) concerns all the inter-nodes $j$ such as $j \leq l$, and ii) that layers expand at the end of the growth cycles (i.e. there is a shift in the indexes of the available quantities of fresh matter). As for leaf and inter-node pith, we define the expansion function of a growth layer: at growth cycle $i$, the expansion function of $l$ th $(l \leq i)$ layer is noted $f_{c}(k)$ with $k=i-l+1$. The quantity $q_{c}(i, l)$ refers to the biomass of $l$ th layer, when plant age is $i$. The layer compartment contains the amount $\left.Q_{c}(i)\right)$ of fresh matter. At cycle 3 , the recurrence equation is:

$$
\begin{aligned}
& {\left[\begin{array}{l}
q_{c}(3,1) \\
q_{c}(3,2) \\
q_{c}(3,3)
\end{array}\right]=\left[\begin{array}{ccc}
f_{c}(1) & f_{(}(2) f_{c}(3) \\
0 & f_{(}(1) & f_{c}(2) \\
0 & 0 & f_{c}(1)
\end{array}\right] .} \\
& {\left[\begin{array}{crr}
1 & 0 & 0 \\
1-f(1) & 1 & 0 \\
(1-f(1))(1-f(1)-f(2)) & 1-f(1)-f(2) & 1
\end{array}\right] \cdot\left[\begin{array}{c}
Q_{d}(1) \\
Q_{d}(2) \\
Q_{d}(3)
\end{array}\right]}
\end{aligned}
$$

\subsubsection{Summary}

Equations (4-6) can be generalized to $N$ growth cycles: the dimension of the system increases of one unit for each new growth cycle. When fresh matter produced at each growth cycle is known (i.e. the set $Q(0), Q(1), Q(2), \ldots, Q(N)$ for a plant of age $N$ ), the model hierarchically allocates this matter among the various organs, according to the strength of the compartment sinks and to the organ expansion functions.

\subsection{Geometry of organs}

\subsubsection{Leaf geometry}

Fresh matter allocated to a leaf is divided into two parts, i.e. to the blade and to the petiole, according to the proportions $c_{l}$ and $1-c_{l}$. The contour of the leaf is arbitrary, while its thickness $(\varepsilon)$ is supposed to be constant. The quantity of fresh matter in the leaf is $q_{a}$. The area of the blade is thus:

$$
S_{a}=c_{l} \cdot \frac{q_{a}}{\varepsilon}
$$

For the petiole, we assume a cylinder shape and an allometric relationship between its length and its cross-sectional area: 


$$
l_{p}, \sigma_{p}=b_{p} \cdot\left|\left(1-c_{l}\right) q_{a}\right|^{\alpha}
$$

where $b_{p}$ and $\alpha$ are scale and shape parameters: $b_{p}$ is related to the thinness of the inter-node, while $\alpha$ controls the behaviour of the ratio $l_{p} / \sigma_{p}$ when petiole biomass (or volume) increases. Particular values of $\alpha$ are:

- $\alpha=0: l_{p} / \sigma_{p}$ is constant whatever the volume of the inter-node;

- $\alpha=1: l_{p} / \sigma_{p}$ is proportional to the volume of the inter-node, which means that $\sigma$ is constant;

$-\alpha=-1: l_{p} / \sigma_{p}$ is inversely proportional to the volume of the inter-node, which means that $l$ is constant;

$-\alpha=-1 / 3: l_{p} / \sqrt{\sigma_{p}}$ is constant, which means that the ratio between the length and the diameter of the cylinder is constant.

When plant age is $i$, the geometry of the petiole of the $j$ th leaf is:

$$
\left\{\begin{array}{l}
l_{p}(i, j)=\sqrt{b_{p}} \cdot\left(\left(1-c_{l}\right) \cdot q_{a}(i, j)\right)^{(1+\alpha) / 2} \\
\sigma_{p}(i, j)=\sqrt{1 / b_{p}} \cdot\left(\left(1-c_{l}\right) \cdot q_{a}(i, j)\right)^{(1-\alpha) / 2}
\end{array}\right.
$$

\subsubsection{Inter-node geometry}

We have to consider two phenomena: the primary and the secondary growth. Indeed, the fresh matter comes from both the inter-node compartment and the layer compartment.

3.6.2.1. Primary growth. The pith is somehow hidden below the external layers. In the model, the pith grows in length and in diameter during a few cycles. As for the petiole, the geometric shape of the pith is a cylinder. Let $q_{e}$ be the fresh matter allocated to a given inter-node, $b_{e}$ and $\beta$ be the scale and shape coefficients of the cylinder. For the $j$ th inter-node at plant age $i$, the length and diameter of the pith are then:

$$
\left\{\begin{array}{l}
l_{e}(i, j)=\sqrt{b_{e}} \cdot q_{e}(i, j)^{(1+\beta) / 2} \\
\sigma_{e}(i, j)=\sqrt{1 / b_{e}} \cdot q_{e}(i, j)^{(1-\beta) / 2}
\end{array}\right.
$$

3.6.2.2. Secondary growth. At each cycle, a new layer is added to the stem so that the external diameter of the inter-nodes grows. The distribution of the fresh matter is supposed to be uniform along the stem. When plant age is $i$, the cross-sectional area of $l$ th layer is therefore:

$$
s_{c}(i, l)=\frac{q_{c}(i, l)}{h(i, l)}
$$

where $h(i, l)$ is the length of the stem up to the top of $l$ th inter-node at plant age $i(1 \leq l \leq i)$. This formula takes the delayed expansion of the existing inter-nodes into account through $h(i, l)$. It is therefore possible to monitor the diameter increment of the stem. When plant age is $i$, the radius of the stem at inter-node $j$ is:

$$
r_{c}(i, j)=\left(\frac{\sigma_{d}(i, j)+\Sigma_{l=j}^{i} s_{d}(i, l)}{\pi}\right)^{1 / 2}
$$

\subsection{Growth simulation algorithm}

In this model, we only consider the aerial system and assume that the biomass of the root system is proportional to the biomass of the aerial part throughout growth. We define $T$ as the number of growth cycles that the plant undergoes.

'Seed biomass' $(Q(0))$ is the initial condition of the iterative growth process. For each subsequent cycle, leaves and inter-nodes are created and expand at the beginning of growth cycles, whereas growth layers (as well as fruits, if they were included in the model, which would be straightforward) are laid down at the end of the cycles. The simulation procedure can therefore be summarized as follows (see table I for the list of the 15 parameters of the model):

- fresh matter is allocated to leaves and internodes for their primary growth, according to the available reserves and to the relative strength of 
the compartment sinks: creation of a new metamer, expansion of the existing metamers;

- the geometry of organs is updated according to their allometric rules and their computed volumes;

- plant architecture is constructed and its hydraulic resistance is computed from the leaves geometry;

- water transpiration of the current growth cycle is computed and converted into fresh matter (using constant WUE) which is added to the previous reserves:

- available fresh matter (from the reserves) is allocated to the layers according to their compartment sink: creation of a new layer, expansion of the existing layers;

- remaining fresh matter is stored for the following cycle.

\section{Main results}

\section{on the single stem cotton-tree}

\subsection{Calibration of the parameters on the cotton plant}

The measurements carried out at the end of the growing season are the cumulative output of plant functioning and development over several months. These data thus constituted targets for the calibration of the theory: plants simulated with AMAPpara should actually match the observations as well as possible. Each of the cotton plants observed in the field was used for model calibration. In this paper, we report the case of only one of them. The other plants from the experiment all yielded similar results [4].

The parameters of the model can be divided into two groups: those which can directly be estimated from measured variables and allometric relationships, and those which are 'hidden' because they are involved in the cumulative process described by the recurrence formulas. For the latter, we need special computational algorithms to calculate their values.

\subsubsection{Estimation of parameters directly linked to plant measurements}

Seven out of the 15 parameters required by AMApara were directly estimated from empirical allometric relationships.

The relative density of the organs was estimated by comparing volumes and weights. The mean val-

Table I. Parameters of the AMAPpara model: meaning and estimated value.

\begin{tabular}{|c|c|c|}
\hline Name & Meaning & Value \\
\hline$Q(0)$ & volume of the seed that initiates the growth process & $Q(0)=51(\mathrm{~g})$ \\
\hline $\begin{array}{l}p_{a}, p_{p}, p_{c} \\
\text { (unitless) }\end{array}$ & $\begin{array}{l}\text { relative strength of the sinks of the leaf, inter-node, and layer compartments } \\
\left(p_{a}+p_{e}+p_{c}=1\right)\end{array}$ & $\begin{array}{l}p_{a}=0.51 \\
p_{e}=0.08 \\
p_{c}=0.41\end{array}$ \\
\hline $\begin{array}{l}b n_{a}, b n_{e}, b n_{c} \\
\left(0<b n^{c}<1\right) \\
\text { (unitless) }\end{array}$ & $\begin{array}{l}\text { organ (leaf, inter-node and layer) expansion functions were modelled } \\
\text { by negative binomial laws truncated at } 12 \text { cycles. The second parameter of } \\
\text { the negative binomial laws was set to } 2 \text {. }\end{array}$ & $\begin{array}{l}b n_{a}=0.86 \\
b n_{e}=0.55 \\
b n_{c}=0.14\end{array}$ \\
\hline$t_{a}$ & life span of the leaves. & 12 (growth cycle) \\
\hline $\begin{array}{l}b_{p}, \alpha \\
\text { (unitless) }\end{array}$ & allometric parameters for petiole shape (cylinder shape) & $\begin{array}{r}b_{p}=68.6 \\
\alpha=0\end{array}$ \\
\hline $\begin{array}{l}b_{e}, \beta \\
\text { (unitless) }\end{array}$ & allometric parameters for inter-node shape (cylinder shape) & $\begin{array}{r}b_{e}=24 \\
\alpha=-0.33\end{array}$ \\
\hline$\varepsilon$ & thickness of leaf blade (lamina) & $\beta=0.043(\mathrm{~cm})$ \\
\hline $\begin{array}{l}\rho_{a 1} \\
\text { (unitless) }\end{array}$ & hydraulic resistivity of leaf blade set for an arbitrary value of the ETP: $E=1000$ & $\rho_{a 1}=770000$ \\
\hline $\begin{array}{l}\rho_{a 2} \\
\text { (unitless) }\end{array}$ & hydraulic resistivity of leaf petiole, set for an arbitrary value of the ETP: $E=1000$ & $\rho_{a 2}=0.96$ \\
\hline
\end{tabular}


ues were $1.06 \mathrm{~g} . \mathrm{cm}^{-3}$ for inter-nodes and $0.96 \mathrm{~g} . \mathrm{cm}^{-3}$ for petioles, these values are close to the hypothetical value of $1.0 \mathrm{~g} . \mathrm{cm}^{-3}$.

For the blade, the relationship between weight $(P)$ and area $(S)$ is linear during leaf expansion and is independent of leaf rank (figure 4): $S=0.043 \cdot P$. We set leaf blade thickness to $e=0.043 \mathrm{~cm}$. Note that we did not measure $e$ and that this parameter is only a scaling coefficient.

The relationship between blade weight (Pblade) and petiole weight (Ppet) of leaves at varying stages of expansion was also linear (figure 5): Pblade $=4.03 \cdot$ Ppet .

Allometric relation between leaf petiole length (Lpet) and leaf cross-sectional area (Spet) was well adjusted by: Lpet $=68.6 \cdot$ Spet $^{1.04}$. This gave: $b_{p}=68.6$ and $\alpha \approx 0$. Thus, petiole length was nearly proportional to the value of the petiole crosssectional area: the ratio of these two values was thus constant and the resistance of the petiole did not depend on its volume.

The allometric relationship between the length (Lintern) and diameter (Dintern) of the pith was studied on the last four inter-nodes, for which the contribution of layers to the total diameter was still low. We obtained: Linter $n=24$. Dintern, which gave: $b_{e}=24$ and $\beta=-0.33$.

The weight of the fresh matter of the whole plant was $1116 \mathrm{~g}$. The total weight of the leaves was $593 \mathrm{~g}$. This gave a first estimate of leaf sink strength as: $p_{a}=593 / 1116=0.53$. Unfortunately, the total weight of the layers could not be distinguished from the total weight of the inter-nodes, because the internal boundary of the pith is not clear.

We observed that leaves were efficient on approximately 12 cycles. Independent leaf pruning experiments showed that there was no difference between plants that retained all their leaves and plants that had kept 15 leaves, whereas the plants with 15 leaves were bigger than those with only 10 leaves. The efficient life span of leaves was therefore set to 12 cycles.

\subsubsection{Estimation of the hidden parameters}

The eight hidden parameters were estimated in order to fit the model predictions to the target

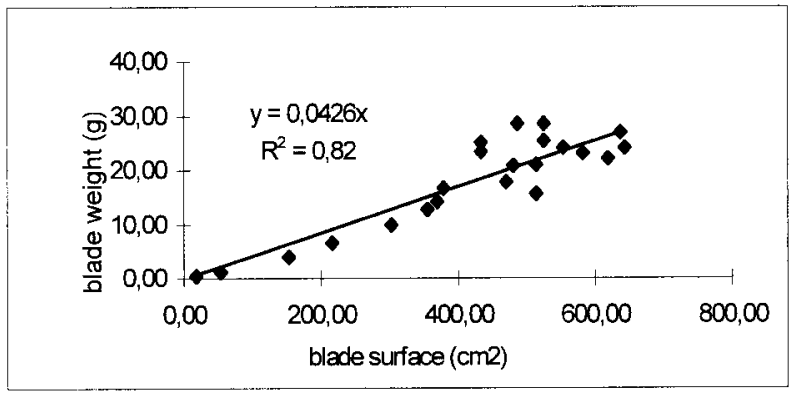

Figure 4. Relationship between leaf blade weight and leaf blade surface in cotton plants.

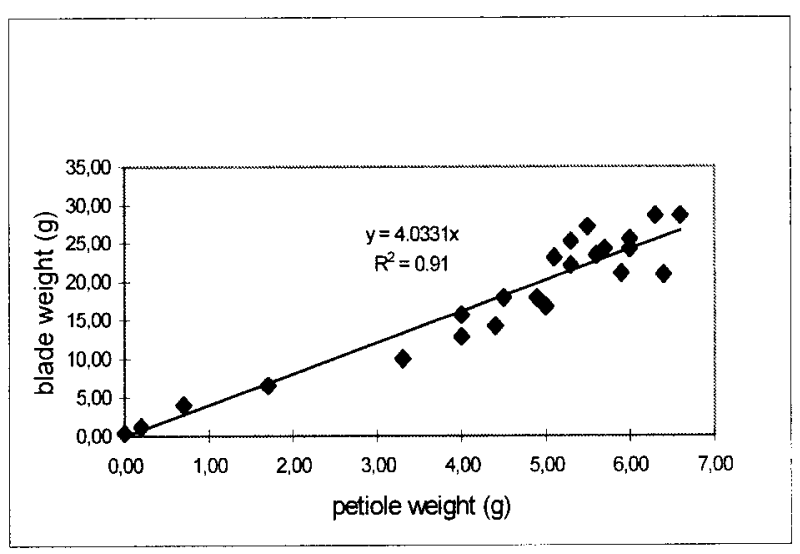

Figure 5. Relationship between leaf blade weight and petiole weight in the leaves of the cotton plant.

experimental data. These data can be summarized into five profiles (blade area, petiole and inter-node length and diameter), which describe the variation in organ size along the stem. More than 100 experimental values were available in order to estimate the eight parameters, thus providing enough degrees of freedom to calibrate the model.

Because of the high number of parameters and of the recurrent nature of the model, we chose to develop a special algorithm for parameter fitting, the so-called 'simulated annealing method' [4]. The advantage of this technique is that it can explore a large range of values for each parameter. Its drawbacks are that a long computing time is required to reach a suitable solution, that convergence towards the solution is slow and that it is difficult to suitably tune the algorithm. There are alternative methods which might converge quicker, 
provided we have good initial (guess) estimates of the parameters. The classical minimization algorithms associated with the least squares criterion are such methods: they are being investigated in order to obtain a better accuracy for the estimation of the hidden parameters. Estimating the parameters of such a model is indeed a complicated mathematical issue by itself. Anyway simulated annealing gave satisfactory preliminary results, both in terms of stability for a given plant and in terms of consistency across plants.

Although leaf sink strength had been directly measured (see above: $p_{a}=0.53$ ), sink strengths were estimated jointly for the three compartments (leaves, inter-nodes, layers). $E$ being arbitrarily set to 1000 (see section 1.3), the estimated values of petiole and leaf resistivity should be considered with caution: their magnitude is biased (i.e. it is proportional to $E$ ), but their ratio is well estimated. Estimated values of the parameters are given in table 1 .

The strength of the leaf sink compartment can be estimated by two techniques: in fact the two estimated values were close to each other $(0.51$ versus 0.53 ). These values indicate that fresh matter was almost equally shared between leaves and inter-nodes (pith and layers).

An enormous difference is observed between blade and petiole resistivity. In fact, if we take organ geometry into account, the estimated values of petiole and blade resistance was of the same order of magnitude. For a mature leaf: i) blade has a thickness of ca. $0.043 \mathrm{~cm}$ and a surface area of ca. $500 \mathrm{~cm}^{2}$; thus, blade resistance is $R_{a 1}=770000 \cdot 0.043 / 500 \approx 66$; ii) petiole diameter is ca. $0.5 \mathrm{~cm}$ and petiole length is ca. $20 \mathrm{~cm}$ so that its resistance is $R_{a 2}=0.96 \cdot 20 /\left(3.14 \cdot 0.25^{2}\right) \approx 98$. The estimate of blade resistance is nearly as large as the estimate of petiole resistance.

As already mentioned, the ratio of blade and leaf resistance does not depend on the arbitrary value chosen for $E$. When the leaf is younger, blade resistance is approximately 1500 , whereas petiole resistance is stable around 100 (because the petiole allometry makes the ratio $l / \sigma$ stable). For young leaves the whole leaf resistance is therefore in its

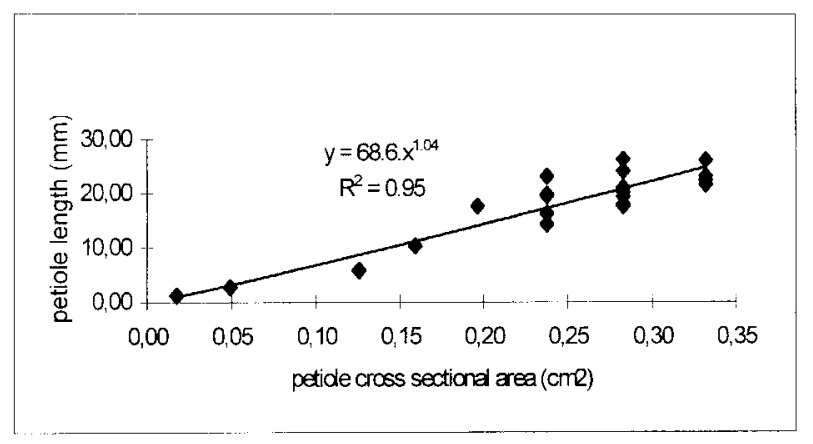

Figure 6. Relationship between petiole cross-sectional area and petiole length.

blade, while petiole resistance becomes more important for mature leaves.

\subsection{Comparison between the original and the simulated data}

In figure 7, we present the results for one of the single stemmed cotton plants (the other pruned plants gave similar results). The target files are the five vertical profiles already mentioned. Although the quality of the adjustment given by the simulated annealing technique could be improved (see above), the fit is fairly good and the residuals are small, especially if we consider that the plants grew in the field, not in a controlled greenhouse or chamber.

These complex profiles are the integrated output of the recurrent functioning of the plant over consecutive growth cycles, so that there is no simple canonical equation to describe their shape. There is indeed a continuous feedback between plant functioning and plant architecture.

On the histogram of the inter-node length, four principal zones, corresponding to typical development stages, are clearly visible along the stem:

- ranks 1-6 are strongly influenced by the initial conditions (seed weight): organs remain small because the amount of available fresh matter is small and the duration of organ expansion limited;

- ranks 7-17 exhibit a sharp increase in the size of organs: inter-nodes and leaves become bigger; 

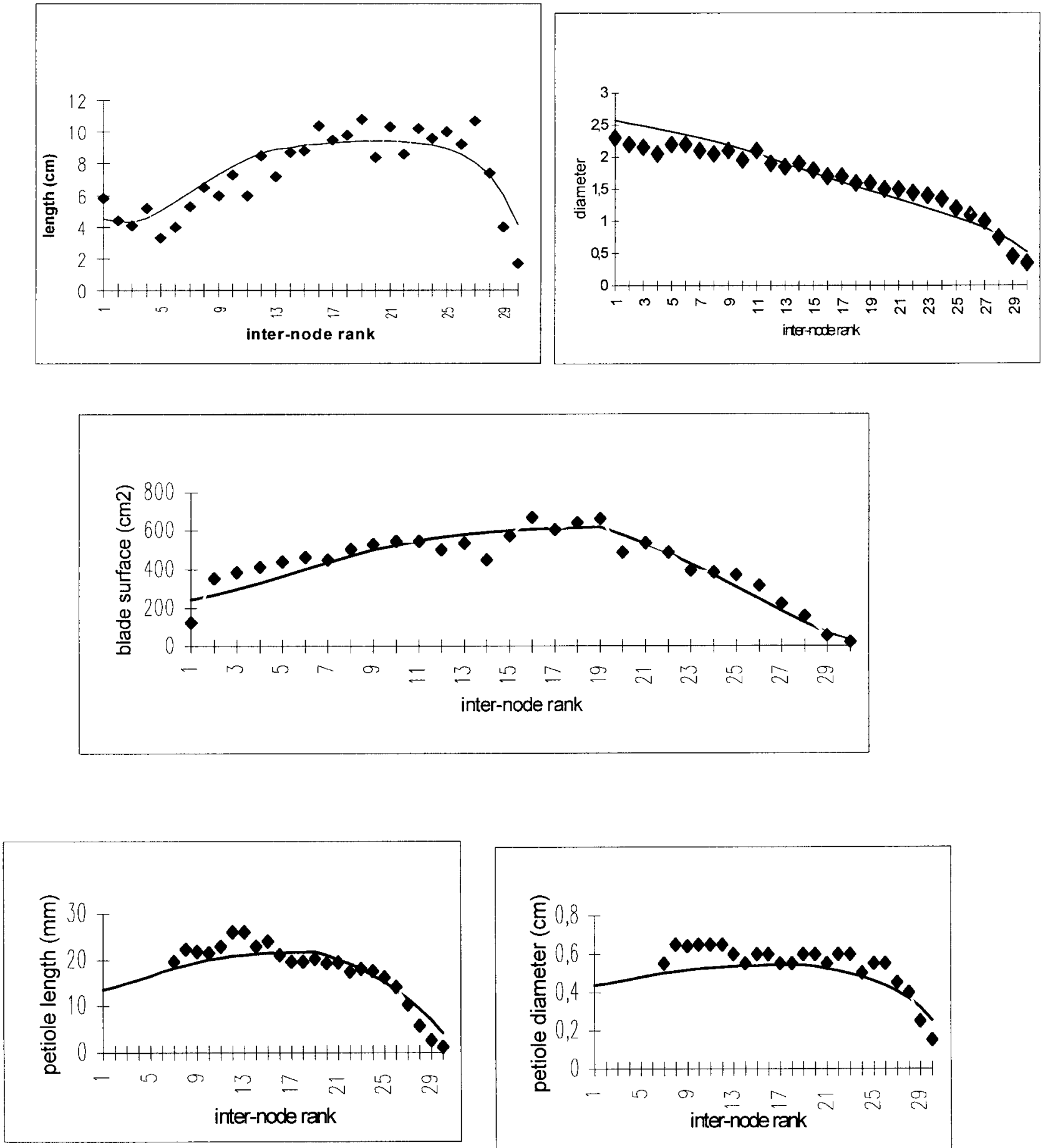

Figure 7. Comparison between the simulated and observed organ sizes of the cotton plant. - Observed values; - predicted values. 
- ranks 18-26 correspond to a stabilization of the growth process that becomes linear: the final size of the organs appears to be stable;

- ranks 27-30 at the tip of the stem are characterized by a decrease in the size of organs, which is due to the fact that these organs have not yet fully expanded (i.e. had the plant not been cut, they would have continued to grow).

Since inter-node expansion is limited to four cycles, these zones are quite visible for inter-node length. For leaves this is less clear because leaf expansion lasts much longer (i.e. about 12 growth cycles). Regarding stem diameter, nothing is visible, but the final conic shape is the result of both pith expansion and of layer stacking at each cycle.

\section{Simulation of plant architecture}

This model was computerized into a generic software that can be used to simulate $3 \mathrm{D}$ virtual cotton plants, according to the values of the previous parameters: the phyllotaxy, branching angle of the leaves and the size of the organs are predicted. The final shape of the real (figure I) and the simulated plant are quite similar (figure 8). Note that in AMAPpara plant geometry is computed and not plated (as in the AMAPsim software [2]).

Figure 8 shows four stages of development at 15 , 20,25 and 30 cycles. We cannot be sure that the intermediate stages are correct. The simulation is

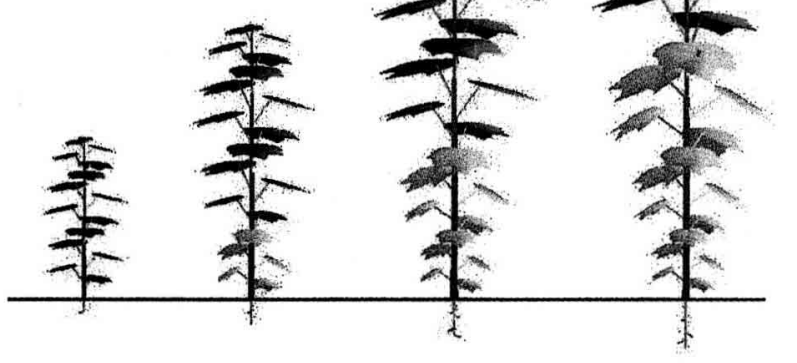

Figure 8. Visualization of 3D simulated cotton plants at 15 , 20,25 and 30 cycles of growth. indeed based on the assumption that the plant underwent average climatic conditions during its growth. However, Chemouny [4] has shown that different climatic growth conditions can produce a fairly similar final plant architecture.

The size of the 15-cycle-aged plant is less than half the size of the plant when it reaches the 30th cycle, whereas the topological structure is exactly half. This is because biomass increment depends on the total leaf area, which itself depends on both the number of leaves and on their individual size.

Theoretically, AMAPpara can support environmental changes from one cycle to the other (e.g. modifications of $E$ ), so that there is a possibility for monitoring plant growth in variable climatic conditions at the cycle level (but not within a cycle). For doing so, it would nevertheless be necessary to establish experimental relationships between light and temperature, on one side, $E$ and resistivity parameters, on the other side.

The model can also support pruning or any artificial modification of plant topology or geometry. Figure 9 shows that simulated pruned and unpruned plants had a qualitative behaviour similar to that of their real counterparts (figure l): the simulated unpruned plant is shorter with smaller organs, but its total biomass is higher than that of the simulated pruned plant; this may be explained by the fact that the amount of biomass per metamer is larger for the pruned plant, for this plant does not divide up its fresh matter production into many competing buds.

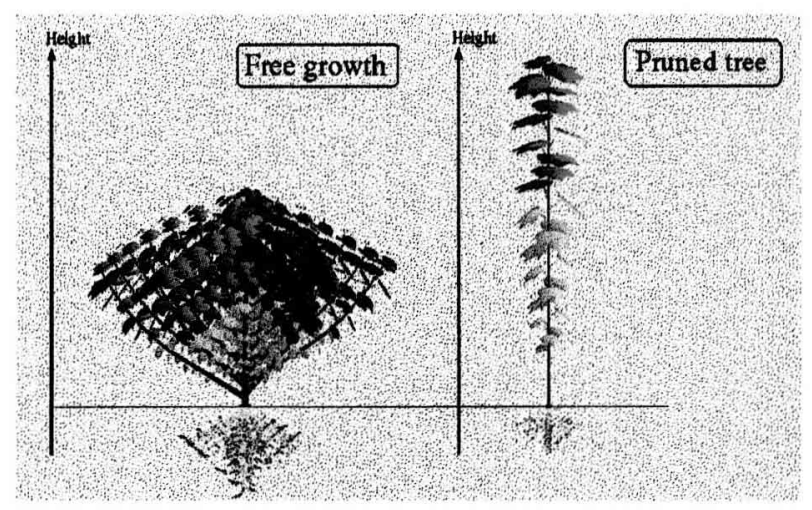

Figure 9. Simulation of branch pruning on the growth of a virtual cotton plant. 


\section{Conclusion}

Earlier models of plant growth and plant architecture developed at Cirad were based on the study of the topological structure of the plant, and focused on the way to dynamically simulate it [2]. Geometry was plated on this structure according to direct field measurements, with the aim of obtaining satisfactory 3D plant mock-ups. Such simulators consisted in dressing plant topology with an empirical geometry. In these models, plant development was already controlled by the concept of growth cycle, which can be viewed as a time scheduler for the creation of new metamers by the buds. A given plant architecture was thus analysed as if it were made up of a finite number of growth cycles without explicit references to real time. Probabilities could also be associated with bud functioning (growth, death and branching) at different levels of the architecture. For example, the AMAPmod software [8] allows these probabilities to be directly estimated from field observations of plant architecture. As a consequence, it is possible to analyse the architecture of a given plant directly as the result of a regular growth process divided into a number of growth cycles. This approach is useful for studying plants, whose past growth conditions are unknown, which is a fairly common situation.

Real time can be introduced into such models thanks to the strong links that exist between the temperature (i.e. thermic time defined by the sum of temperatures) and growth rate (i.e. expressed in terms of number of growth cycles per time unit) [12]. The duration of each cycle can thus be estimated from field, greenhouse or chamber experiments. But even in this case, there is no feedback between plant growth and plant architecture: plant topology and organs are produced according to a pure morphogenetic model, but they do not play any functional role by themselves. Such models can neither be used for simulating the influence of technical interventions, nor for predicting the impact of changing ecological conditions: the plants are not sensitive to their environment.

The goal of this paper was to demonstrate that it is possible i) to extend the concept of growth cycle to the production of biomass, and ii) to calibrate a process-based architectural growth model using experimental data. We assumed that, under normal conditions, the amount of energy provided during a growth cycle is fairly constant, so that the environmental trends disappear when growth cycle is considered rather than time (figure 2). Random oscillations around the mean were observed on experimental data, but their effect was dampened by the expansion of the organs that lasted over several cycles. Observed cotton plants had a quite homogeneous architecture; the evolution of metamer size within the plant was regular and the residual noise was low.

We then assumed that, in the absence of hydric stress conditions, the production of biomass during a growth cycle is directly under the control of water transpiration, i.e. that it depends on WUE. As a consequence, we defined the hydraulic architecture of the plant. For cotton, we supposed that the hydraulic resistance was concentrated in the leaves and was shared between blade and petiole, which are disposed in series and whose resistance depends on their geometry and on a resistivity factor.

The architectural point of view turned out to be quite efficient and an explicit reference to the exact values of the physical environmental parameters was not necessary for calibrating the model. Indeed, we assumed that biomass increment was proportional to a constant parameter representing the potential of fresh matter production during a growth cycle (parameter $E$ ) and inversely proportional to plant hydraulic resistance. An arbitrary value was set for $E$, which acts as a common scaling factor for blade and petiole resistivity. The other parameters of AMAPpara control seed reserves, and organ sinks, allometry, expansion and resistivity. These parameters are sufficient to describe the allocation of the fresh matter during a growth cycle and to iteratively construct plant geometry.

Half of the parameters could be directly obtained from simple plant measurements. The others had to be estimated by more sophisticated minimization techniques (i.e. simulated annealing, 
non-linear least squares). Model calibration was satisfactory: the vertical profiles of organ size along the stem were well predicted.

As leaf area plays an explicit role in this model, it is possible to modify either plant architecture or environmental conditions during the growth. Feedback interactions between plant structure and growth are automatically represented through recurrent growth equations. It is therefore now possible to link the computation of plant geometry to the computation of its topological structure. The present version of AMAPpara [16] can only simulate simple plant architectures, but it relies on the same basic assumptions as AMAPsim [2], which is already able to simulate the topology of complex plants. It is thus possible to contemplate simulating virtual plants reactive to their environment, and experimentally calibrating such models. Agronomic applications based on plant growth monitoring and optimizing at the cycle level is becoming a goal for the near future.

Acknowledgement: The authors are grateful to Pascal Clouvel from the Department of Annual Crops at Cirad, for his friendly scientific and technical support.

\section{References}

[1] Baker D.N., Lambert J.R., Mc Kinion J.M., Gossym: a simulator of cotton growth and yield, Technical Bulletin no.1089, South-Carolina Agricultural Experiment Station, Clemson University, Clemson, 1983, p. 134.

[2] Barczi J.-F., Reffye (de) Ph., Caraglio Y., Essai sur l'identification et la mise en oeuvre des paramètres nécessaires à la simulation d'une architecture végétale: le logiciel AMAPsim, in: Bouchon J., Reffye (de) P., Barthélémy D. (Eds.), Modélisation et simulation de l'architecture des végétaux, Inra-Editions, Versailles, 1997, pp. 255-423.

[3] Blaise F., Barczi, Jaeger M., Dinouard P., de Reffye P., Simulation of the growth of plants. Modelling of metamorphosis and spatial interactions in architecture and development of plants, in: Kunii T.L., Luciano A. (Eds.), Cyberworld, Springer-Verlag, Tokyo, 1998, pp. 81-109.

[4] Chemouny S., Estimation des paramètres du modèle de croissance et d'architecture végétale AMAPpara. Application au cotonnier taillé, DEA, université des sciences et techniques du Languedoc, Montpellier, 1997.

[5] Dauzat J., Eroy N.M., Simulating light regime and intercrop yields in coconut based farming systems, European Society for Agronomy, 7-11 July, Wageningen, The Netherlands, 1996, p.16.
[6] Fournier C., Andrieu, B,. Utilisation de l'approche L-Système pour la modélisation architecturale du développement du maïs, Actes du séminaire sur la modélisation architecturale, Paris 10-12 march 1997, Département de Bioclimatologie Inra, 1997, pp. 203-211.

[7] Gary C., Barczi J.F., Bertin N., Tchamitchian M., Simulation interactive de la croissance d'une plante de tomate en fonction de son environnement et de sa conduite (modèle TOMGRO), in: Bouchon J. (Ed.), Architecture des arbres fruitiers et forestiers, Inra-Editions, Versailles, 1995, pp. 333-343.

[8] Godin C., Guédon Y., Costes E., Caraglio Y. Measuring and analysing plants with the AMAPmod software, in: Michalewicz M.T. (Ed.), Advances in Computational Life Sciences: Plants to Ecosystems, CSIRO, Australia, 1997, pp. 53-84.

[9] Kurth W., Morphological models of plant growth: possibilities and ecological relevance, Ecol. Model. 75-76 (1994) 299-308.

[10] Linterman B., Deussen O., Interactive models of plants, IEEE Comput. Graphics Applic. 19 (1999) 56-65.

[11] Perttunen J., Sievänen R., Nikinmaa E., Salminen H., Saarenmaa H., Väkevä J., LIGNUM: a tree model based on simple structural units, Ann. Bot. 77 (1996) 87-98.

[12] Poisson C., Rey H., Caraglio Y., Modélisation de l'architecture et de la croissance de cinq espèces du genre Nicotiana, Ann. Tabac, section 2, vol. 2 (1997) 37-54.

[13] Prusinkiewicz P., Hanan J., Hammel M., Mech R., L-systems: from the theory to visual models of plants, in: Michalewicz M.T. (Ed.), Advances in Computational Life Sciences: Plants to Ecosystems, CSIRO, Australia, 1997, pp. 1-27.

[14] Rapidel B., Étude expérimentale et simulation des transferts hydriques dans les plantes individuelles: application au caféier (Coffea arabica L.), thèse, université de Montpellier II, France, 1995.

[15] Reffye (de) P., Houllier F., Blaise F., Barthélémy D., Dauzat J., Auclair D., A model simulating above- and below- ground tree architecture with agroforestry applications, Agrofor. Syst. 30 (1995) 175-197.

[16] Reffye (de) P., Fourcaud T., Blaise F., Barthélémy D., Houllier F., A functional model of tree growth and tree architecture, Silva fennica 31 (1997) 297-311.

[17] Reffye (de) P., Houllier F., Modelling plant growth and architecture: Some recent advances and applications to agronomy and forestry, Curr. Sci. 73 (1997) 984-992.

[18] Ryan M.G.,Yoder B.Y., Hydraulic limits to tree height and tree growth, Bioscience 47 (1997) 235-242.

[19] Williams J.R., Jones C.A., Kiniry J.R., Spanel D.A., The EPIC crop growth model, Trans. Am. Soc. Agric. Eng. 32 (1989) 497-511. 